\title{
EL PRINCIPIO DE ASISTENCIA HUMANITARIA Y LA MUJER DESPLAZADA EN COLOMBIA
}

\section{Myriam Constanza Caicedo Enríquez}

\section{Resumen:}

El presente artículo describe los lineamientos de la comunidad internacional respecto al desplazamiento interno y el Principio de Asistencia Humanitaria. Además, consolida la posición de la Corte Constitucional, a partir de sus pronunciamientos, particularmente de la sentencia T-025 de 2004 y el Auto 092 de 2008, con énfasis en la atención y protección a la mujer desplazada y se resalta su aporte para el diseño de la política estatal del desplazado.

Titulo: El principio de asistencia humanitaria y la mujer desplazada en Colombia

Palabras claves: Desplazamiento forzado, Mujeres desplazadas, Asistencia humanitaria, Principios Rectores, Comunidad Internacional, Corte Constitucional.

\section{Summary}

The current article describes the international community alignments regarding internal displacement and the Humanitarian Assistance Principle. Also, consolidates

- Abogada de la Universidad Santo Tomás y docente Programa de Derecho. Universidad Mariana. Pasto. E-mail: myriam85@hotmail.com 
the Constitutional Court position, based on their statements, particularly form the T025 sentence from 2004 and the Auto 092 from 2008, emphasizing on the protection and care of the woman under forced displacement and highlighting their contribution with the design of the displacement government policy.

Title: The Humanitarian Assistance Principle and the Colombian Woman under forced displacement.

Keywords: Forced Displacement, women under displacement, humanitarian assistance, Guiding Principles, International Community, Constitutional Court. 


\section{INTRODUCCIÓN}

La población desplazada en Colombia, constituye un fenómeno social muy sensible ante la comunidad internacional, ocasionado por el conflicto interno que lo ha llevado a una violencia política en los últimos 50 años, catastrófica en términos de pérdida de vidas, aparición de nuevas formas de violencia, recrudecimiento del conflicto armando, deterioro de la cultura democrática, altos niveles de corrupción y menoscabo de los derechos humanos. En este sentido, Colombia padece una crisis de grandes proporciones en lo social, político y económico.

El presente artículo muestra el papel del Estado colombiano frente a los Principios rectores de la Asistencia humanitaria a la población desplazada, particularmente, a la mujer víctima del desplazamiento forzado. La mujer por su condición de género y el predominio de una cultura patriarcal en la sociedad colombiana, está más expuesta a padecer los avatares de este fenómeno.

Tradicionalmente la mujer cumple varios roles: madre, hija y hermana; en la mayoría de los casos debe responsabilizarse por sus hijos, hermanos y padres; situación que la hace vulnerable frente a la sociedad, por ello, la necesidad de

comprender desde la academia cómo afronta el Estado, haciendo uso de la normativa sobre los derechos humanos, tan importante deber, el de brindarle asistencia humanitaria que la comunidad internacional recomienda. 
Así pues, el artículo sintetiza los parámetros de la comunidad internacional enfocados a la asistencia humanitaria y el desplazamiento forzado de la mujer y muestra la importancia de la Corte Constitucional que a través de sus pronunciamientos ha ido constituyendo la orientación para las políticas estales frente a la problemática.

Para el logro del objetivo, se desarrollan cuatro partes; en la primera, consideraciones iniciales, se describen los antecedentes de los principios rectores del desplazamiento y el marco jurídico internacional, donde se señala la vinculación de los tratados internacionales al marco jurídico nacional; en la segunda, sistematización del Derecho Internacional Humanitario y de los Principios rectores del desplazamiento, se ilustra la incorporación de los principios en el derecho interno, destacando la acción activa de la Corte Constitucional, en sus decisiones trascendentales del Auto 092 de 2008 y la Sentencia T-025 de 2004; en la tercera parte, reflexiones de diferentes actores frente a la política pública de desplazamiento, se complementa con visiones tanto internacionales como nacionales y académicas sobre la política pública del desplazamiento, se resalta como común denominador la baja acción estatal y; por último, en la influencia de la Corte Constitucional, se destaca el accionar del Estado a partir del evidente, oportuno y acertado papel de la Corte Constitucional. 
Finalmente se presentan historias de vida y conclusiones, donde se puede apreciar la fuerza del fenómeno del desplazamiento, su efecto en las personas y se invita a continuar ampliando la investigación en tópicos como la aplicación de la política de desplazamiento.

\section{EL PRINCIPIO DE ASISTENCIA HUMANITARIA Y LA MUJER DESPLAZADA EN COLOMBIA}

\section{CONSIDERACIONES INICIALES}

\subsection{Los principios de protección a los desplazados}

Los principios de protección a los desplazados, son expresión de la comunidad internacional para contribuir a establecer una base jurídica que vele por las personas que en un país no están involucradas en conflictos internos. En ese orden de ideas, en este trabajo se inicia presentando una visión de su consolidación, articulados con el marco jurídico internacional y finalmente su incorporación en Colombia.

El representante del Secretario General de las Naciones Unidas [ONU], desarrolló el plan de acción propuesto en el Estudio comprensivo denominado Compilation and Analysis of Legal Norms (1995), en el cual se reúnen un trabajo de un jurista austriaco y otro estadounidense; a partir de éstos, definió las necesidades de los 
desplazados internos y realizó la revisión de las normas de Derechos Humanos, Derecho Internacional Humanitario y Derecho de los Refugiados, aplicables en situaciones de disturbios interiores, conflicto armado no internacional y conflicto internacional. Con este marco, el documento estableció la existencia de normas dentro del Derecho Internacional que protegen a los desplazados durante el desplazamiento y en situaciones de reubicación y retorno.

Además, en la segunda parte de la "Compilación y análisis de normas" (1995), se abordó el problema de protección frente al desplazamiento y el derecho a no ser desplazado. Los dos documentos en conjunto fueron la base para un tercero, en el que se publicaron los Principios Rectores sobre desplazamiento Interno [Report Addendum. Guidins Principles] que, actualmente, constituyen el eje central del Derecho Internacional en materia de desplazados (Vidal Lopez, 2007).

Dichos principios fueron presentados a la Comisión Internacional de Derechos Humanos [CIDH] en 1998, y fue hasta el año 2005 cuando los Estados los reconocen como un "marco internacional de importancia para proteger a las personas desplazadas dentro de sus países" (Vidal Lopez, 2007, p. ). Los mismos reafirman y reflejan la normativa internacional vigente en materia de derechos humanos y derecho humanitario relacionada con los desplazados internos. Su objeto es servir de norma internacional para orientar a los gobiernos y demás actores en el suministro 
de asistencia y protección a la población desplazada, identificando sus derechos y garantías durante toda la etapa de desplazamiento (Instituto Brookings, 1999).

Así mismo, en la teoría del derecho internacional, los Estados se obligan, mediante tratados internacionales, costumbres, principios generales de derecho, jurisprudencia y doctrina (estas dos últimas como fuentes auxiliares), a la protección de la población en situación de desplazamiento. Sin embargo, cuando el tema que lo ocupa se relaciona con la dignidad humana, su responsabilidad aumenta y se hace un llamado a normas imperativas. Es así como los Estados deben proteger la dignidad como un hecho de obligatorio cumplimiento.

Además, los gobiernos prohíben la intervención en situaciones de conflicto dentro de la jurisdicción de cualquier Estado, procurando protección y asistencia humanitaria a todos los ciudadanos, incluyendo, en especial, a personas desplazadas internamente, constituyendo esto un deber y una responsabilidad primordial del Estado. La Asamblea General de Naciones Unidas, en algunas situaciones, ha reafirmado lo siguiente: "La soberanía de Estados afectados y su labor principal está en la iniciación, organización, coordinación e implementación de la asistencia humanitaria dentro de sus respectivos territorios" (ONU, 1990, p.).

Es de resaltar que la asistencia humanitaria, según Hardcastle y Chua (1998), hace referencia "a una amplia variedad de actividades internacionales, incluida la 
ayuda a las víctimas de conflictos y de intervenciones armadas para reinstaurar la democracia", que se basa en principios como la humanidad, imparcialidad, neutralidad e independencia (ACNUR, 2010).

De igual manera, instrumentos como la Convención Americana de Derechos Humanos (año) hace referencia a derechos tales como: el de la vida, debido proceso y movilidad, entre otros, permitiendo así al Sistema Interamericano de Derechos Humanos conocer sobre las violaciones de los derechos de los desplazados y la asistencia humanitaria brindada en el país. Es importante tener en cuenta que Colombia ratificó y se adhirió a este tratado el 28 de Mayo de 1973, siendo entonces vinculante.

\subsection{Marco jurídico de la comunidad internacional frente al desplazamiento forzado interno y su incorporación en Colombia}

Respecto al Marco jurídico internacional, se debe destacar que se constituye en actos jurídicos, que tienen en cuenta los principios rectores de desplazamiento y se componen por parámetros tendientes a proteger los derechos humanos de una población en situación de vulnerabilidad, que deben ser asumidos e implementados por los órganos estatales. 
Varios tratados internacionales prohíben y hasta entienden el desplazamiento forzado como un crimen internacional. Dentro de estos tratados se encuentran: el Pacto Internacional de los Derechos Civiles y Políticos de la Organización de Naciones Unidas [ONU], aprobado, mediante depósito del instrumento de ratificación, el 29 de octubre de 1969, el cual entró en vigencia en Colombia el 23 de marzo de 1976 ; el Pacto Internacional de los Derechos Económicos, Sociales y Culturales, que Colombia aprobó por ley 74 de 1968 y lo ratificó, al igual que el Protocolo Facultativo, el 29 de octubre de 1969; el Estatuto de Roma aprobado el 10 de diciembre de 1998 y ratificado el 5 de agosto de 2002; además, la Convención de Belem Do Para que se firmó en 1994 y se aprobó mediante ley 248 de 1995, fecha en que comenzó a regir, y, en ese mismo orden, los Convenios de Ginebra.

Para el caso del desplazamiento forzado interno, en orden a dar cumplimiento a las obligaciones básicas, la Comisión Interamericana, la Organización Africana, la Organización para la Seguridad y Cooperación en Europa y la Organización del Commonwealth, recomiendan la utilización de lo manifestado en la Resolución A/RES/64/162 de 17 de marzo de 2010 y los "Principios rectores del Desplazamiento Interno ó Principios Deng" (ONU, 1998; 2010); así, los tratados de derechos humanos que conforman el marco jurídico comportan para el Estado tres obligaciones básicas: 
1. Respetar los derechos reconocidos en dichos tratados; 2. Garantizar el goce y pleno ejercicio de los derechos protegidos a las personas que se encuentran bajo su jurisdicción; y 3. Adoptar las medidas necesarias para hacer efectivos los derechos protegidos. (Martín, Rodríguez y Guevara, 2004, p. ).

La jurisprudencia internacional ha sostenido que las obligaciones de respetar los derechos humanos y de garantizar su goce y pleno ejercicio, constituyen el fundamento genérico de la protección de los derechos humanos. "Los principios consagran elementos esenciales para el enfrentamiento del problema del desplazamiento, sintetizan la doctrina internacional tomada en materia de derechos de la población desplazada y contiene una construcción política integral con enfoque de derechos" (Uprimmy y Saffon, 2006, p. ).

\section{SISTEMATIZACIÒN DEL DERECHO INTERNACIONAL HUMANITARIO Y LOS PRINCIPIOS RECTORES DEL DESPLAZAMIENTO}

\subsection{Incorporación de los principios en el Derecho Interno de Colombia}

El artículo 93 de la Constitución Política de Colombia de 1991, en cuanto a la incorporación de los principios mencionados prescribe que: "prevalecerán en el ordenamiento interno de los tratados aceptados por Colombia sobre derechos humanos y derecho internacional humanitario". A su vez, el artículo 94 establece que: "La enunciación de los derechos y garantías contenidos en la Constitución y en 
los convenios internacionales vigentes, no debe entenderse como negación de otros que, siendo inherentes a la persona humana, no figuren expresamente en ellos", lo que hace referencia, directamente, a la aceptación para Colombia del lus Cogens o Normas de imperativo cumplimiento.

Las otras normas que tienen un tratamiento especial, son las del Derecho Internacional Humanitario [DIH], en virtud del artículo 214-2 de la Carta, que prescribe: "En todo caso se respetarán las normas del Derecho Internacional". Al respecto, la Corte Constitucional según Sentencia C-156 de 1999, ha interpretado que el DIH es aplicable en Colombia en cualquier tiempo, sin que sea relevante si se ha declarado un estado de conmoción interior o hay un estado de normalidad. En virtud de la misma norma, hay incorporación automática de la totalidad del DIH al ordenamiento interno colombiano. "La Corte le atribuye el carácter de ius cogens". (Vidal López, 2007, p. )

Por su parte, los Principios rectores se han incorporado a través de una línea jurisprudencial que viene desde el año 2000. Entre las sentencias que otorgan el carácter de integrantes del Bloque de Constitucionalidad están:

SU-1150 de 2000, T-1635 de 2000, T-327 de 2001, T-098 de 2002, T-268 de 2003, T-419 de 2003, T-602 de 2003 y T-025 de 2004. En estas, se han incorporado los Principios como una herramienta interpretativa y algunas de sus normas se han vinculado de forma directa en el bloque de constitucionalidad. (Piedad y Daniel, 2008, p. ). 


\subsection{Puesta en marcha de la Obligación para Colombia}

¿Cuál es el objetivo de los principios? Para responder a este interrogante, la Corte constitucional en sentencia T-025 de 2004, dice:

Buscan atender las necesidades particulares de las personas en situación de desplazamiento, identificando los derechos y garantías que son relevantes tanto para la prevención del fenómeno, como la protección de quienes lo sufren, tanto durante el desplazamiento como al momento de reintegrarse 0 restablecerse en otro entorno.

El desarrollo que traen los principios conlleva, según la misma sentencia, una definición de los desplazados internos como: "aquellas personas o grupos de personas que han sido forzadas $u$ obligadas a huir $\mathrm{o}$ abandonar sus hogares $\mathrm{o}$ sus sitios de residencia habitual sin cruzar una frontera internacional".

En el año 2004, la Corte Constitucional, acumuló 108 acciones de tutela interpuestas por 1150 núcleos familiares pertenecientes a la población desplazada, con un promedio de cuatro personas por núcleo, y compuestas principalmente por mujeres cabezas de familia, personas de la tercera edad, niñas y niños, además de algunas personas indígenas. (Mujer, 2008, p. )

La Corte, evidenció la grave situación de vulnerabilidad de las personas desplazadas y solicitó a las instituciones gubernamentales que presentaran su diagnóstico sobre 
el estado de esta población y cuál era la respuesta del Estado colombiano. Hizo, además, consultas con otras entidades para informarse sobre esta situación; concluyendo que, no sólo las personas que presentaron la acción de tutela debían ser susceptibles de protección, sino todas aquellas que se encuentren en la misma situación fáctica.

Teniendo en cuenta esto, las mujeres encontraron que la tutela respondía a la petición de muchas de ellas que se encontraban preocupadas por sus familias y su propia integridad, facilitando el proceso de fortalecimiento organizativo que les brindó una herramienta para discutir sus problemáticas en el marco Constitucional. Se ve, además, para el año 2006 la necesidad de adoptar una política pública más eficaz con enfoque diferencial en cuanto a edad y género.

Por otra parte, a partir del análisis de la información recibida por las organizaciones de mujeres, las organizaciones sociales, las entidades del Sistema Nacional de Atención Integral a la Población Desplazada, el estudio de casos presentados ante la Corporación y el informe presentado por la Comisión Interamericana de Derechos Humanos, a propósito de la visita a Colombia, en junio de 2005, de la Relatora sobre los Derechos de las Mujeres, relacionada en el documento: Las mujeres frente a la violencia y la discriminación derivadas del conflicto armado en Colombia, la Corte reconoce el impacto desproporcionado, en términos cuantitativos y cualitativos, del conflicto armado interno y del desplazamiento forzado sobre las mujeres colombianas (Mujer, 2008). 
Si bien la Corte, es consecuente con la problemática del desplazamiento y de su impacto en la población vulnerable, también en ese sentido contribuyen los aportes de la Comisión Interamericana de Derechos Humanos por el grave impacto que el conflicto armado en Colombia ha generado, al respecto dice:

La población civil no-combatiente ha sido severamente afectada por el conflicto, sobre todo los grupos que están expuestos a mayor riesgo, como las mujeres, los niños y niñas, los pueblos indígenas, y las comunidades afro colombianas, así como los líderes sociales y organizaciones que asumen la defensa de sus derechos.

Es evidente que la Corte asume el informe de la CIDH para sustentar su posición jurídica y plantear medidas de política pública al Estado, pues da un reconocimiento a más de la mitad de la población afectada por el desplazamiento en Colombia, que representan las mujeres, como las víctimas con mayor desprotección.

Así el papel de la Corte es llamar al Estado a implementar medidas integrales y de manera especial para buscar erradicar patrones socioculturales discriminatorios en razón de sexo, raza, etnia y clase social, y a tomar en cuenta estas diferencias en el desarrollo de políticas públicas. 


\subsection{Implicaciones del Auto 092 de 2008 Corte Constitucional}

En Colombia la atención a la población desplazada tiene su principal soporte tanto en los reclamos de las personas afectadas, los requerimientos de la comunidad internacional como en la jurisprudencia de la Corte Constitucional.

Lo anterior, converge en tres aspectos que resultan fundamentales y que han sido tratados en la Sentencia T-025 de 2004 y en el Auto 092 de 2008, estos son: El reconocimiento de las mujeres como sujeto político, víctimas y sujetos de derechos, el reconocimiento de prácticas de violencia sistemática contra las mujeres en el marco del conflicto armado y la validación de la importancia de la democracia y los mecanismos de control y división del poder. El Auto 092 de 2008 señala:

Que la respuesta estatal frente a estas situaciones ha sido insuficiente y que los elementos existentes de la política pública de atención al desplazamiento forzado dejan vacíos críticos que resultan en una situación de total desamparo de las mujeres desplazadas ante las autoridades obligadas a protegerlas.

Frente a lo anterior, se declara en el mismo Auto, que las autoridades deben actuar a fin de prevenir el impacto desproporcionado del desplazamiento sobre las mujeres, y garantizar el goce efectivo de sus derechos fundamentales. 


\subsection{Formulación de una política pública}

La Corte, con el Auto 092 de seguimiento, no sólo diagnosticó la situación, sino que se centró en proponer una formulación de política pública, a raíz de la situación de violación masiva de los derechos fundamentales de la población femenina desplazada. Lo más importante de este Auto, es que trata el elemento diferenciador de género y, por lo tanto, enumera las problemáticas que enfrentan las mujeres en condición de desplazamiento.

Hasta el momento de la expedición del Auto, no existían medidas ni legales ni de política pública que atendieran las necesidades específicas de las mujeres en condición de desplazamiento, y, por ende, se violaron sus derechos de forma sistemática y masiva. En resumen, no existían acciones estatales encaminadas a la protección de sus derechos fundamentales.

Entonces, de acuerdo con el pronunciamiento de la Corte, es teniendo en cuenta esos factores de género que se deben diseñar, desarrollar y poner en funcionamiento los diferentes programas. En el Auto se denota que, para ese momento, las medidas adoptadas por el gobierno no eran específicas -aprobación de normas, atención en comisarías de familia, centros de atención a víctimas, 
Implementación de proyectos interinstitucionales-; esas medidas, incluso, se puede manifestar que su aplicabilidad se da para población en general. No se hacía diferenciación sobre la atención a las mujeres en condición de desplazamiento.

\subsection{El Principio de Asistencia Humanitaria en Colombia}

En correspondencia con el Auto 092, está lo relacionado con la asistencia humanitaria, para esto se parte anotando que las obligaciones estatales no sólo deben versar sobre las prescripciones nacionales y ser apegadas a la letra en cuanto a lo que las políticas públicas establezcan, sino que: "deben ser armónicas con las prescripciones internacionales" (ONU, 2000) ${ }^{1}$.

Además, el principio rector de desplazamiento tercero (ONU, año), específicamente en su numeral 1, señala que: "Las autoridades nacionales tienen la obligación y la responsabilidad primarias de proporcionar protección y asistencia humanitaria a los desplazados internos que se encuentren en el ámbito de su jurisdicción". Este como se describió antes, incorporado a la legislación nacional, se desarrolla en la sentencia T - 025 de 2004 que manifiesta:

\footnotetext{
${ }^{1}$ Resolución 1325 de 2000 del Consejo de Seguridad de la ONU, que estipula dos puntos principales: 1. Mecanismos institucionales eficaces y, 2. Las partes en un conflicto armado deben adoptar medidas especiales para proteger a las mujeres y las niñas de la violencia por razón de género.
} 
El derecho básico a recibir asistencia humanitaria para cubrir necesidades del mínimo vital, se extiende hasta el momento en el cual la circunstancia en cuestión sea superada.

De esto, se puede extraer que para las mujeres es un avance y como se anoto antes, se aprecia, entonces, la consecuencia armónica de la posición de la Corte con respecto a la mujer desde el punto de vista objetivo. Tal tratamiento de la asistencia humanitaria, se desprende de su naturaleza como cabeza de familia, pues, finalmente, es en la mujer que recae la atención de los hijos, de los niños y ancianos. Esta realidad es evidente en el país, donde a la mujer se le ha asignado roles complementarios del hogar, constituyéndose esto como una característica cultural.

\section{REFLEXIONES DE DIFERENTES ACTORES FRENTE A LA POLÍTICA PÚBLICA DEL DESPLAZAMIENTO}

\subsection{Visiones sobre la Política pública del desplazamiento en Colombia}

Los organismos internacionales y las organizaciones no gubernamentales se han constituido como el ojo crítico en materia de aplicación de la política pública de atención a la situación de desplazamiento; son aquellos quienes proponen las recomendaciones que debe ejecutar el Estado, todas ellas dentro del marco jurídico y normativo descrito. Entre estas organizaciones se encuentran: 
3.1.1 El Comité Internacional de la Cruz Roja [CICR]. Éste no siempre utiliza el mismo método de atención para las situaciones de desplazamiento; entre sus procedimientos de trabajo se encuentran: 1. Persuasión: consiste en diálogos con las autoridades estatales en orden a lograr un convencimiento de negociación. 2. Denuncia: método que se encuentra destinado a ejercer presión sobre la autoridad o apremiar para dejar que se continúe cometiendo la violación a un derecho. 3. Sustitución: cuando se actúa en lugar de la autoridad para poner fin a una violación o para ayudar a las víctimas de ésta -apoyo indirecto o institucional-.

Sin perjuicio del método utilizado por los Organismos Internacionales y Organizaciones no gubernamentales, los desplazamientos internos, según la Comunidad Internacional, se consideran como uno de los asuntos humanitarios pendientes que merecen una mayor atención, y el desarrollo de un ámbito de protección de las necesidades de seguridad, subsistencia y dignidad en las diferentes categorías de desplazados, como: mujeres, niños, adultos mayores e indígenas.

En tal sentido, la protección "abarca todas las actividades tendientes a conseguir el pleno respeto de los derechos de las personas, de conformidad con la letra y el espíritu de la normativa pertinente" (apellido de autor, año, p. ), es decir, la protección brindada no se puede encontrar por fuera del marco jurídico previamente expuesto, toda vez que el desplazamiento es la "consecuencia de una violación 
deliberada o no de las normas jurídicas y responsabilidad de afrontarlos que recae sobre las autoridades nacionales" (CICR, 2009, p. ).

3.1.2 Organización de las Naciones Unidas [ONU]. La primera recomendación para el gobierno frente a este fenómeno, hecha por la ONU, es el establecimiento de un mecanismo de coordinación dentro del gobierno central, el cual será el encargado de atender la política de atención a la población desplazada; frente a esto, se habla de la creación de un Comité Permanente entre Organismos, el que se constituiría en una red superior sobre los desplazamientos internos que ayude a evaluar la calidad de las acciones humanitarias; organismos que, a su vez, se encargarían de realizar recomendaciones a los diferentes entes que atienden a la población desplazada.

La segunda recomendación, para el caso colombiano, hace referencia a quienes se encuentran encargados de realizar las políticas públicas de atención en cuanto a asistencia humanitaria, como órganos, principalmente, de carácter político; sin embargo, la ONU ha dicho que uno de los puntos débiles, del sistema de atención, es que no se percibe "una política estatal y una estrategia material integral que traduzca el plan normativo en programas concretos".

Adicional a lo anterior, se encuentra una limitada asignación y ejecución de recursos; no existe la asunción de papeles preventivos por parte de los Comité locales, así como también, no existe un sistema de alertas tempranas con cobertura 
nacional. Dentro de las falencias que recalca el Alto Comisionado de la ONU, se resalta la falta de respuesta de la fuerza pública. Aunque estos aspectos no hacen parte integral de la política pública en materia de asistencia humanitaria, se encuentran estrechamente ligados a las obligaciones estatales, de respetar y garantizar los derechos de las personas en situación de desplazamiento forzado.

3.1.3 Posiciones académicas. De acuerdo con el estudio realizado por el Instituto Latinoamericano de Servicios Legales Alternativos [ILSA], titulado: Desplazamiento y retorno, balance de una política, publicado en el 2006, se plantean las siguientes recomendaciones referentes al tema de estudio:

La asistencia humanitaria debe prolongarse por el tiempo que dure la situación de desplazamiento y siempre debe ser oportuna y completa (alimentación, kit doméstico, de aseo, de cocina, alojamiento), de acuerdo a las necesidades de cada familia o persona... La asistencia no debe descuidar el apoyo sicosocial, la asesoría jurídica, la documentación, los servicios de salud y educación, y los esfuerzos por una inserción en el mercado nacional. Es necesario explorar alternativas que surtan de trabajo temporal a miembros de familias desplazadas. Estas recomendaciones han de ser implementadas en su mayor parte por los Comités Locales y Departamentales de Atención Integral a la Población Desplazada. (Piedad y Daniel, 2008) 


\subsection{El Registro como desafío de la Política pública}

De acuerdo con diferentes Organismos Internacionales, una de las principales falencias es el registro.

La polémica sobre las cifras del desplazamiento, desde la perspectiva metodológica, gira en torno a la relación existente entre dicha definición conceptual y la forma en que es aproximada mediante los instrumentos técnicos utilizados por los diferentes sistemas de seguimiento a la población desplazada.

La postura más radical asumida por el Gobierno nacional, ha sido la de identificar su instrumento técnico: el Registro Único de Población Desplazada (RUPD), como un indicador directo y exacto de la definición conceptual, es decir, afirmar que la dimensión del desplazamiento forzado es exactamente ofrecida por el RUPD. Poco a poco, más que todo mediante los autos de la Corte Constitucional relacionados con el seguimiento de la política de atención a desplazados, se ha enfatizado la existencia de subregistros y de problemas de caracterización de la población desplazada. Generando esto, que el presupuesto destinado a este fenómeno, no corresponda a la dimensión real del problema. (Rodríguez, 2010, p. 38; Auto 011, 2009) 
Ahora bien, además del problema primario mencionado, se encuentra que la caracterización no permite tener una información socioeconómica y demográfica suficiente. "La información del RUPD... padece de un defecto de caracterización, pues los individuos registrados no han sido apropiadamente analizados" (Rodríguez Garavito, 2010).

Por su parte, la ONU ha mencionado que no existe un mecanismo que facilite el acceso a los programas, siendo, por ende, un obstáculo al acceso de la asistencia humanitaria por parte de la población desplazada. Las causas identificadas para que el registro de la población desplazada sea débil, han sido: la interpretación restrictiva de normas, la aplicación del principio de extemporaneidad (principio incompatible con los Principios rectores de los desplazamientos Internos), y existe una restricción de contextos, es decir, únicamente son considerados desplazados aquellos que se encuentran en dicha situación a causa del conflicto armado; pero, personas que salen de sus tierras como consecuencia de la ejecución de megaproyectos y de acciones estatales como las fumigaciones no se encuentran, según el gobierno colombiano, dentro de la categoría de desplazado; sumado a esto, la falta de conocimiento de la norma tal como se puede observar en las historias anexas.

Los anteriores aspectos, además, son identificados como un no respeto por los derechos básicos, introduciendo de ésta forma requisitos discriminatorios para el acceso a la asistencia humanitaria en la primera etapa del desplazamiento. Aunado a 
lo anterior, una vez la población tiene acceso a la asistencia humanitaria, se encuentra "un desequilibrio entre la Asistencia Humanitaria de Emergencia [AHE], de tres meses, y el periodo de tiempo más extenso que necesiten los desplazados para ser autosuficientes" ${ }^{2}$

Las recomendaciones respecto del problema del registro, dadas por la Organización de Naciones Unidas (2006), son:

1. Aplicación de enfoques diferenciales a los diferentes grupos poblacionales tanto para el registro como para la prestación de la Asistencia Humanitaria de Emergencia. 2. Aplicación de una política integral en materia de género. 3 . Protección de las mujeres con respecto a la violencia intrafamiliar, tráfico de personas y efectos derivados del conflicto. 4. Promoción de los proyectos de asistencia humanitaria de emergencia, alojamiento, apoyo financiero, sanidad, y asistencia psicológica durante la etapa de emergencia posterior al desplazamiento. 5. Cooperación entre el Estado y los entes gubernamentales encargados de brindar la asistencia humanitaria. 6. Capacitación en derechos humanos y técnicas de fomento en la autosuficiencia. 7. El Estado Colombiano

\footnotetext{
${ }^{2}$ Frente a este punto cabe hacer la aclaración, que las falencias de la política pública en atención a la población desplazada, en especial en cuanto a la Atención Humanitaria de Emergencia, ha sido tema de debate jurisprudencial por parte de la Corte Constitucional, en donde se estableció que, contrario a como en un inicio lo estableció la ley 387, la AHE no puede ser limitada únicamente a un periodo de tres meses, toda vez que el nivel de vulnerabilidad de esta población (como las mujeres madres cabeza de hogar) merece especial atención y ayuda por parte de los entes gubernamentales, razón por la que la AHE es prorrogable de forma indefinida hasta tanto se verifique que la población se puede sostener de forma autosuficiente.
} 
debe ejecutar los programas de asistencia humanitaria y de desarrollo en coordinación con entidades internacionales con experiencia en el tema, asegurando que se facilite el alojamiento adecuado, se cumpla con las reglas básicas en materia de salud e higiene y se respeten las unidades familiares. 8. Lograr una mayor coordinación entre las autoridades nacionales y locales. 9. Establecimiento de una mayor presencia internacional, especialmente sobre el terreno. 10. Agilizar y suprimir los requisitos del registro que resulten contrarios a las disposiciones del Derecho Internacional. 11. Atención específica a las necesidades particulares de las mujeres y niños. 12. Se asegure el acceso a los programas estatales a través del registro incluyendo la atención humanitaria de emergencia de conformidad con las normas internacionales, las internas y las constitucionales. 13. Continuar con el apoyo de la asistencia humanitaria por parte de las Organizaciones no Gubernamentales, en especial a las mujeres y los niños.

El método de trabajo del Departamento para la Prosperidad Social (año), antigua Acción Social, es: "apoyarse en Organizaciones como la Cruz Roja para brindar la asistencia humanitaria en los desplazamientos masivos de 50 o más personas, asistencia humanitaria que resulta de calidad para los desplazados". Sin embargo, los desplazamientos de menos de cincuenta personas son asistidas por otras organizaciones que delegan sus funciones a subcontratistas, quienes no tienen la infraestructura y los recursos para atender el fenómeno, quedando estas 
asistencias, como ellos mencionan: en el papel. Es por ello que en estos desplazamientos no se puede descartar el buen recibimiento de la Asistencia Humanitaria de Emergencia por los proyectos internacionales (Refugees International, 2007).

\subsection{Detección de las dificultades por parte de los Órganos Nacionales}

La Corte Constitucional en Auto 011 del 2009 ha mencionado que las siguientes son las falencias de la política de atención a la población en situación de desplazamiento:

a) No existe información oportuna y completa de sus derechos; b) el sistema de información no contiene la totalidad de ayudas; c) existe un subregistro; d) no hay registro de las ayudas provistas; e) no es sensible a las necesidades de los desplazados; y f) la política se encuentra formulada sobre presupuestos que no se corresponden con la verdadera dimensión del problema que se pretende atender.

Así mismo, frente a las anteriores falencias las Corte identificó, en primer término, el derecho de habeas data que tienen las personas desplazadas, en lo que denominó en habeas data aditivo, lo que consistiría en el "derecho de inclusión de los datos personales del sujeto interesado en el banco de datos de los programas" (Corte Constitucional, año). Por lo tanto, dentro de esta órbita los derechos de la población desplazada serían: a) El derecho a figurar con su identidad clara en el 
Registro Único de Población Desplazada y otros sistemas. Para la Corte, la recomendación sería llenar los vacíos y actualizar los sistemas; b) el sistema de registro completo permite una efectiva protección en cualquier política pública; y c) generaría una responsabilidad por la discrepancia entre las bases de datos.

Por lo pronto, las recomendaciones concretas a efectos de superar las falencias identificadas son: a) Frente al problema del subregistro: Establecimiento de un cronograma para registrar a la población desplazada antes del 2000, y el registro de los niños que nazcan posteriormente al registro del desplazamiento; b) frente a la discrepancia entre las bases de datos que registran a la población desplazada: Debe existir una comunicación de la información del Registro Único de la Población Desplazada y las entidades prestadoras del servicio; c) se debe crear un sistema armónico que promueva resultados relevantes para el goce de los derechos, lo que se constituye, a su vez, en dar información útil y suficiente.

Finalmente, un estudio académico realizado (escribir titulo del estudio y año) reconoce que la política pública de atención a la población desplazada es deficiente; sin embargo, CODHES ha dicho que el componente más exitoso de la política, es la Asistencia Humanitaria en porcentajes equivalente al $35 \%$ y el $40 \%$, aspecto que no resulta de alivio para la garantía del acceso a la atención humanitaria, toda vez que el momento de mayor vulnerabilidad es el ocurrido luego del desplazamiento, y el 
porcentaje presentado demuestra que aún así existe deficiencia en la atención humanitaria.

Por lo anterior, el profesor Uprimny en el artículo mencionado recomienda: "La comunidad internacional debe ser un ojo crítico en la insistencia de protección de todas las etapas, verificando la atención de la población desplazada" (Uprimmy y Saffon, 2006, p. ).

Con respecto a la Comunidad internacional y el control que esta ejerce dentro del proceso de atención a la población desplazada, resulta necesaria en orden a mejorar la política pública. Así mismo, la colaboración que suministra es clave frente a la prestación de la Asistencia Humanitaria, lo que no significa un desentendimiento por parte del Estado de sus obligaciones internacionales, sino que se debe generar y mejorar la simbiosis que existe entre el Estado y los organismos de la Comunidad Internacional que, actualmente, intervienen y presentan sus opiniones.

\subsection{Acciones Estatales}

Es importante recordar que las mujeres, como grupo de población desplazada, se constituyen como las más afectadas, principalmente, por el tema de inequidad que existe entre hombres y mujeres; sufriendo, así mismo, un impacto altamente negativo a causa del desplazamiento forzado. Para contrarrestar las discriminaciones a las que se enfrentan las mujeres, se requiere del diseño, evaluación y ejecución de 
políticas públicas, para que los planes y programas beneficien de forma equitativa a las mujeres y a los hombres. "El derecho de las personas desplazadas internamente de solicitar y recibir protección y asistencia humanitaria de autoridades nacionales es la labor principal del Estado" (Oficina de coordinación de asuntos humanitarios ONU).

En este sentido, en Colombia las acciones estatales que buscan favorecer la situación de la población en desplazamiento, vinieron a configurarse con la ley 387 de 1997, la cual, a largo plazo, no resultó ser suficiente para atender la situación de esta población, por lo que la Corte Constitucional, a raíz de las constantes violaciones a los derechos y acciones judiciales encaminadas a buscar la protección y garantía de dichos derechos, adoptó una posición de "excepcionalidad", en orden a proteger esos derechos afectados, y, entre ellos, los de las mujeres en particular.

Por consiguiente, es a partir de la sentencia T-025 de 2004, que se produce un fenómeno, considerado híbrido por la doctrina, en cuanto al diseño de las políticas públicas, en especial, la encaminada a la atención de la población en condición de desplazamiento, y los pronunciamientos y diseños judiciales de estas políticas realizadas por la Corte, resulta una combinación entre lo jurídico y lo económico; al respecto se ha dicho:

...el impacto del caso T-025 sobre las políticas públicas sobre el desplazamiento..., su incidencia en la situación de la población desplazada. En cuanto al primero de estos impactos, que llamamos el "efecto de políticas públicas", uno de los aspectos más significativos del fallo y los autos es que su 
aparato conceptual y su lenguaje son un híbrido entre: 1) las herramientas jurídicas del derecho constitucional y el derecho de los derechos humanos, y 2) las herramientas de la economía y las políticas públicas.

De las primeras proviene la lógica legal y prescriptiva que lleva a la Corte a concluir la existencia de una violación masiva de derechos y declarar el estado de cosas inconstitucional. De las segundas viene la lógica organizacional y los instrumentos de análisis y medición de políticas con los que la Corte diagnostica las fallas estructurales de la administración pública e impulsa el diseño, la ejecución y la evaluación de nuevas políticas, que incluye un largo y sofisticado proceso de construcción de indicadores de avance o retroceso en la garantía de los derechos de los desplazados. (Rodríguez y Rodríguez, 2010, p. )

La anterior constituye la lógica utilizada al momento de la evaluación de las políticas desarrolladas por el Estado para garantizar y proteger los derechos de la población desplazada, lógica consignada en la sentencia creadora de la línea jurisprudencial sobre desplazamiento forzado.

\section{INFLUENCIA DE LA CORTE CONSTITUCIONAL}

\subsection{El papel activo de la Corte Constitucional}

La Corte Constitucional está asumiendo una función humanitaria frente a las diferentes situaciones de vulnerabilidad de la población desplazada; de manera 
concreta ha entrado a incidir en las políticas sociales, llenando un vacío del Estado que, por consideraciones económicas, no atendía; esto explica el por qué a la Corte le ha correspondido involucrarse en los aspectos económicos como soporte de sus decisiones. De éstas, además, se desprenden los elementos para las políticas públicas, destacando que pone de relieve el papel del desplazado como agente, y, de manera especial, la mujer.

\subsection{Componentes fundamentales de las políticas públicas}

La Corte, mediante el ejercicio de su papel formulador, a través de los diferentes autos de seguimiento, ha puesto en conocimiento cuáles deben ser los elementos de una política pública orientada a la atención de la población desplazada; dichos elementos fueron puestos en conocimiento, no solamente en el auto 092 de 2008, sino en todos aquellos que hacen referencia a un punto de vista de género y especificidad.

En el auto 251 de 2008, la Corte anota, de forma concreta, cuáles son los elementos que una política pública debe tener, partiendo de la especificidad y la divisibilidad, especialmente, para mujeres, niños y ancianos.

\subsection{Política Pública de atención humanitaria}

La política pública, según Bejarano Cepeda (2010) se divide en tres partes, éstas son: 
1. Situación inicial y las órdenes dictadas por la Corte.

Teniendo como base la sentencia T-025 de 2004, se determinó que el derecho a la subsistencia mínima consiste en que las autoridades competentes deben proveer: alimentos esenciales y agua potable, alojamiento y vivienda básica, vestidos apropiados y servicios médicos y sanitarios esenciales.

De acuerdo a lo estipulado, la Atención Humanitaria de Emergencia se otorga durante tres meses, tiempo que no es razonable para poder planificar a corto plazo y acceder a las posibilidades razonables de subsistencia, y puede ser extendida hasta que la situación de urgencia haya pasado y se haya logrado, por parte del desplazado, una estabilidad para conseguir su propia subsistencia.

Ahora ¿quiénes son los titulares de recibir la Atención Humanitaria de Emergencia? La política está orientada hacia dos tipos de desplazados: quienes estén en situación de urgencia extraordinaria, y quienes no estén en condiciones de asumir su auto sostenimiento -mujeres cabeza de familia, niños sin acudientes, adultos mayores-.

2. Diseño de la Política e idoneidad frente a criterios de racionalidad.

De acuerdo con la política diseñada por el Estado, la atención humanitaria "está orientada a garantizar la subsistencia mínima de la población en situación de desplazamiento, en tres momentos: urgencia, emergencia y transición" (DNP), cada 
uno de ellos con características especiales y otorgamiento de acuerdo a los criterios de vulnerabilidad.

3. Herramientas de instrumentalización de la política de Asistencia Humanitaria.

Se debe, no sólo garantizar las respuestas a las peticiones realizadas por parte de la población desplazada, sino que, también, se debe dar atención prioritaria a los grupos de especial protección constitucional, lo cual se configuraría como parte de la garantía de la subsistencia mínima.

\section{CONCLUSIONES}

La comunidad internacional, principalmente el Consejo de Seguridad, Económico y social, Asamblea General y la Coordinación de asuntos humanitarios de la ONU y la Comisión Interamericana de Derechos Humanos (CIDH), han expresado lineamientos teóricos y jurídicos que son expresión de su misión para facilitar a los Estados parámetros que garanticen la protección de los derechos humanos, a la población civil vulnerable en situación de conflictos internos.

Los pronunciamientos teoricos y juridicos corresponden a los principios rectores que versan sobre la protección a la población desplazada, sus derechos fundamentales y medidas de prevención, que son de responsabilidad de los estados. 
En Colombia el desplazamiento viola los derechos humanos de manera sistemàtica a ciertos grupos de personas, entre estos, las mujeres que se encuentran en situación más vulnerable frente a la violencia y discriminación.

La Corte Constitucional ante la situación del conflicto interno colombiano, el fenómeno del desplazamiento y la presión de las personas afectadas como las mujeres mediante la acción de tutela, se ve ante la necesidad objetiva de cumplir su misión de guarda suprema de los derechos fundamentales y de la Constitución Política, en consecuencia se pronuncia de manera activa y oportuna frente al papel que el Estado colombiano debe asumir, particularmente con la sentencia T-025 de 2004, el Auto 092 de 2008 y demás Autos de seguimiento, donde cabe resaltar, que acoge las orientaciones internacionales.

Los pronunciamientos de la comunidad internacional y la Corte Constitucional, han construido un marco teorico y jurídico, que le facilita al Estado el diseño de una política de atención a las víctimas del fenómeno, y además, debido a la condición de la mujer se evidencia su proteccón especial.

Dentro de la sistematización normativa y jurídica se ve la necesidad que el Principio de Asistencia Humanitaria, se entienda como un escenario de complementariedad a una política de restablecimiento de derecho y de estabilización socioeconómica. 
Teniendo en cuenta la complejidad del tema y que en Colombia es tan importante para la convivencia, se requiere ampliar la imvestigación al campo de la ejecución de las políticas y programas que el Estado ejecuta. 


\section{FUENTES Y BIBLIOGRAFÍA}

Doctrina:

Bejarano Cepeda, G. (2010). Derecho a la reparación integral. Cátedra semipresencial "Desplazamiento Forzado en Colombia". Manuscrito no publicado.

Beristain, C. (s.f.). Contenidos y dilemas de la ayuda humanitaria, una perspectiva psicosocial crítica. Recuperado el 11 Marzo de 2011, de http://terranova.uniandes.edu.co/centrodoc/docs/AtenciondeEmergencia/Texto s/Contenidos\%20de\%20la\%20AHE.pdf

Chua, R. J. (1998, 31 de diciembre). Asistencia humanitaria: hacia el derecho de tener acceso a las víctimas de desastres naturales. Revista Internacional de la Cruz Roja. Recuperado de http://www.icrc.org/spa/resources/documents/misc/5tdmnv.htm

Instituto Brookings. (1999). Guia para la aplicación de los principios rectores de los desplazamientos internos. Recuperado el 8 de octubre de 2011, de http://www.acnur.org/biblioteca/pdf/7368.pdf?view=1

Laserna, A. (2005, 5 de Marzo). Asistencia humanitaria en Colombia. En Sens Public. Recuperado el 20 Octubre de 2011, de http://senspublic.org/spip.php?article147\&lang=fr

Martin, C., Rodríguz Pinzón, D., \& Guevara, J. A. (2004). Derecho Internacional de los Derechos Humanos. México: Doctrina Jurídica Contemporánea.

Piedad, C. L., \& Daniel, M. (2008). Desplazamiento y retorno. Balance de una política. Bogotá D.C: ILSA.

Rodríguez Garravito, C. (2010). Más allá del Desplazamiento Políticas, derechos y superación del desplazamiento forzado en Colombia. Bogotá D.C: Uniandes.

Rodríguez, C., \& Rodríguez, F. (2010). Cortes y cambio social, cómo la Corte Constitucional transformó el desplazamiento forzado en Colombia. Bogotá D.C: Colección de Justicia.

Uprimmy, R., \& Saffon, M. P. (2006). Desplazamiento forzado y justicia transicional en Colombia. Bogotá: DJS. 
Vidal López, R. C. (2007). Derecho Global y Despalazamiento Interno. Bogotá D.C: Pontificia Universidad Javeriana.

Organismos internacionales:

ACNUR (2010, 19 de agosto). Celebrar a quienes trabajan ayudando a los demás, recordar a quienes perdieron sus vidas en el intento. Recuperado el 10 de 2011, de http://www.acnur.org/t3/el-acnur/eventos/dia-mundial-humanitario-2010/

Comisión Interamericana de Derechos Humanos. (2006). Las mujeres frente a la violencia y la discriminación derivadas del conflicto armado en Colombia. Recuperado el 20 de octubre de 2010, de http://www.cidh.oas.org/countryrep/ColombiaMujeres06sp/lyll.htm

Comisión Interamericana de Derechos Humanos. (s.f.). Recuperado el 8 de octubre de 2010, de http://www.cidh.oas.org/countryrep/ColombiaMujeres06sp/resumenejecutivo.ht $\mathrm{m}$

Comité Internacional de la Cruz Roja Ginebra. (2009). Protección de los desplazados internos: Concepto y desafíos. International review of the red cross. Naciones Unidas. (11 de Febrero de 1998). Recuperado el 1 julio de 2010, de http://www.hchr.org.co/documentoseinformes/documentos/html/informes/onu/r esdi/E-CN-4-1998-53-ADD-2.html

Consejo Económico y Social de Naciones Unidas. (1998, 11 de febrero). ACNUR. Obtenido de http://www.acnur.org/t3/fileadmin/scripts/doc.php?file=biblioteca/pdf/0022

Naciones Unidas. (1990, 14 de diciembre). Asamblea General. Recuperado el 9 de Octubre de 2011, de http://daccess-ddsny.un.org/doc/RESOLUTION/GEN/NR0/572/63/IMG/NR057263.pdf?OpenEle ment

Naciones Unidas. (2010, 17 de marzo). Asamblea General. Recuperado el 10 de Octubre de 2011, de http://daccess-ddsny.un.org/doc/UNDOC/GEN/N09/471/58/PDF/N0947158.pdf?OpenElement 
Naciones Unidas. (2000, 31 de Octubre). Consejo de Seguridad. Recuperado el 11 de enero de 2011, de http://daccess-ddsny.un.org/doc/UNDOC/GEN/N00/720/21/PDF/N0072021.pdf?OpenElement

Nacions Unidas. (2006). Poner fin a la violencia contra la mujer: de las palabras los hechos. Recuperado el 10 de enero de 2011, de http://www.un.org/womenwatch/daw/vaw/publications/Spanish\%20study.pdf

Oficina de Coordinacion de Asuntos Humanitarios de las Naciones Unidas. (s.f.). Principios Rectores De Los Desplazamientos Internos Recuperado el 8 enero de 2011, de http://www.law.georgetown.edu/idp/spanish/GPSpanish.pdf

Refugees International. (2007). Colombia Falla en Proporcionar asistencia adecuada a la Población Desplazada. [Bulletin]. Washington D.C: Sean Mariano Garcia y Andrea Lari.

United Nations. (1995, 5 de Diciembre). Office of the United Nations High Commissioner for Human Rights. Recuperado el 10 de 2011, de http://www.unhchr.ch/Huridocda/Huridoca.nsf/0/75550ee91a4fb1ff802566cc00 5c2c63?Opendocument

Jurisprudencia:

Corte Constitucional. (1999, marzo 10). Sentencia C-156 de 1999. Recuperado el 1 octubre de 2011, de http://www.observatorioddr.unal.edu.co/ambitojuridico/catalogo_juridicojyp/sent encia_c-156-99.pdf

Corte Constitucional. (2008, abril 14). Auto 092 de 2008. Recuperado el 3 julio de 2010, de http://www.corteconstitucional.gov.co/relatoria/autos/2008/a09208.htm

Corte Constitucional. (2004, enero 22). Anexo 3 Sentencia T-025 de 2004. Recuperado el 8 julio de 2010, de http://www.corteconstitucional.gov.co/relatoria/2004/t-02504\%20anexo\%203.htm

Corte Constitucional. (2004, enero 22). Sentencia T-025 de 2004. Recuperado el 11 de julio de 2010, de http://www.corteconstitucional.gov.co/relatoria/2004/t-02504.htm 
Corte Constitucional. (2009, enero26). Auto 011 de 2009. Recuperado el 2 de octubre de 2010, de http://www.acnur.org/t3/fileadmin/scripts/doc.php?file=biblioteca/pdf/6984

Corte Constitucional. (2008, octubre 6). Auto 251 de 2008. Recuperado el 11 de febrero de 2011, de http://carlosvicentederoux.org/apc-aa files/d1d179e489889931192d79ba046874e0/AUTO\%20251\%20de\%202008.p df

Departamento de Derecho Internacional OEA. (s.f.). Tratados multilaterales. Recuperado el 8 de octubre de 2011, de http://www.oas.org/dil/esp/tratados_B 32_Convencion_Americana_sobre_Derechos_Humanos_firmas.htm

Forzado, C. (2008). VI Informe a la Corte Constitucional. Bogotá D.C.

Instituciones públicas colombianas:

Ley 387 (1997, 18 de julio). Gobierno Nacional. Congreso de Colombia. Ministerio del Interior $y$ de Justicia. http://www.secretariasenado.gov.co/senado/basedoc/ley/1997/ley_0387_1997. $\mathrm{html}$

Ley 248 (1995, 29 de diciembre). Gobierno Nacional. Congreso de Colombia. Ministerio del Interior $y$ de Justicia. En http://www.secretariasenado.gov.co/senado/basedoc/ley/1995/ley_0248_1995. $\mathrm{html}$

Departamento Nacional de Planeación. (SF). Módulo VI: Acciones conducentes a la garantía del goce efectivo de derechos. Recuperado el 20 de enero de 2012, de http://www.dnp.gov.co/LinkClick.aspx?fileticket=G9Hzz_olVaA\%3D\&tabid=129 6

Internacional, C. d. (2012, Junio 12). Coalisiçon por la Corte Penal Internacional. Recuperado el 1 de enero 2012, de http://www.iccnow.org/?mod=country\&iduct=37\&lang=es

Mujer, C. C. (2008, agosto 8). Semanario virtual Caja de Herramientas. Recuperado el 22 agosto de 2010, de http://www.viva.org.co/cajavirtual/svc0122/index\%20\%20pagina\%207.html 
Presidencia República de Colombia. (1988, Octubre 13). Recuperado el 8 de octubre 2011, de ftp://ftp.camara.gov.co/camara/basedoc/decreto/1988/decreto_2110_1988.html REDEPAZ. (s.f.). Plan Nacional de Atención y Reparación Integral a Víctimas. Recuperado el 9 de enero de 2012, de http://www.redepaz.org.co/IMG/pdf/3726_anexo.pdf 


\section{ANEXO}

A

1.- Narración de su historia de vida, de donde es, hace cuando sufrió desplazamiento, tiene conocimiento de los autores, fechas, to que hizo una vez le ocurrió este hecho.

2.-Ante quien ha acudido para obtener ayuda.

3.- Ha recibido la ayuda humanitaria de emergencia? En caso afirmativo, cuantas veces, cuanto tiempo tardó la entrega de esas ayudas, ante quien acudió, quien atendió su solicitud.

Sino to ha recibido cual considera ha sido ta causa que han incidido en este hecho, ya to solicitó? Hace cuanto, que respuesta le han dado?

4.- Cual es su opinión frente a la ayuda recibida, esta de acuerdo? Que mejoria o cambiaría

5.- Como califica la colaboración que brinda el gobiemo para atender su situación?

6.- Que recomendaciones hace a la politica de ayuda de emergencia y ta atención humanitaria 
Soy del Homieipio de Combitara, Vereda de Santa 1 - Ana, tengo 33 años, tengo 5 hijos en e da des de 16 años a 8 años, 4 mujeres y un bomboe. Soy madre cabera de familia, mi compañero me abandonó hace 7 años, acteralmente mis hijos están al cuidado de mimadre.

En el año del zood, en el mes de Agosto, el dia 16 , hubo un énfantamiento del los te la vereda tuvimos gise salir y nos venimos a Pasto, a donde unos fami liares, La situación pconónica era dificil, nos fuimos a la crur raja para quo nos a yu be, y mego on Acción Social, donde los metieron al sistema 4 los car retizaion.

Cono la situación econónica estaba mue dera, y mi compañerome tejó sola, me, fioco po nerme a trabajar y cai en la cáreel a donde esfoy pagandosina condeno de 64 meser de los cuales llevo 2 años fisicos.

2. Ayuda me trevon la cruz Raja y Acción Social, hasta 3 meses con mercados, y en Acción Social 2 veces para artencamiento, Y no más.

3. Solo las veces que anoto en el punto $z$.

42 
21- Porqiae me darcuido en seguir metiento los papelos qia mo solicitaban, porque no habiar quien meagude y o too poorite caíen la carrel camente esto y sola. llo y practiapoyo te nadie.

5 - A peser de que actualmen te no tengo apoyo, la labor de la acción social, es BuenA, porqua me ayo do as,' séa poco, en el mo mento que már 10 necesite, con mi familia.

- Que no se olvide de las personas que como yo, muchas veces por necesi dad se mete encosas gito no lobe, como es llegar a la càre, pro nadie tiene un censo * cuantés de las personas qlap he mos sido clasplazades por el conflic to arma o, estamos en las cárceles pagando delitos.

Seria bieno qub al menos sepan en que condicienos astamos nosotros y nuestoas familices.

Mario Ofelia Rosero

re. 27456491 
1.- Narración de su historia de vida, de donde es, hace cuando sufrió desplazamiento, tiene conocimiento de los autores, fechas, lo que hizo una vez le ocurrió este hecho.

2.-Ante quien ha acudido para obtener ayuda.

3.- Ha recibido ta ayuda humanitaria de emergencia? En caso afirmativo, cuantas veces, cuanto tiempo tardó la entrega de esas ayudas, ante quien acudió, quien atendió su solicitud.

Sino lo ha recibido cual considera ha sido la causa que han incidido en este hecho, ya to solicitó? Hace cuanto, que respuesta le han dado?

4.- Cual es su opinión frente a la ayuda recibida, esta de acuerdo? Que mejoría o cambiaría

5.- Como califica la colaboración que brinda el gobierno para atender su situación?

6.- Que recomendaciones hace a ta política de ayuda de emergencia y la atención humanitaria 
1. Yo me llamo Blanca Lur Diar Pay, sou de Samomegfo, teng 43 años, soy madre cabera te familia, tengo 3 hijitos, de 22, 16 y 21 años. Tengo 2 niños entencrdos de 8 y 9 años quse quederon huerfanos, parqias a sos papas los mató la guerrilla.

Cuando se dió el derplaramiento, hace 7 años, wivamo con mi mamá y mifamilia en la Vereda SANDE, en Samamiego, cuendo lleyaron la Guemilla y nos amenuraion y nos dijeron qite salieramos qive porqle los trabajadores eran paracos, allé matason a 2 personas, y salimos con loqias teniamos. puesto, yo con mi hijor cogí para Barbacoory ni mamà y hermanos para el Putomago.

En la Vereda Buena Vista de Barbacoas, Aonde me

- fir con mifamilia, tambier llogó la guernillay nató a 7 trabajaldores de la finca don de trabajarba y me tocó salir corriendo con mitamitia a Riacurte, conde me tocó torberjas en to que fuera, porque no tenia nada) y caí con drogery ahora estoy en la caircel, hace 20 meses, con una condena de taños.

Mis hijos están donde una tamiliar en Burbacoas. yestán mal, me preace pa que los mayo res cozen malos cominos, no quero que terminen como go en una cairne!. Asi se tiro uno la vide.

45 
2- Lo primero quo hicimos fué ir a hablar con los Gukernadores indigenas, peso no noscay uderon en nada, eso Fieé en Ricaurte.

Nate me dió razón a quen acudir pera ques meayuden, mi la Alealdia, is la personesia, si nodie.

3. No he recibido ninguna ayuda, solo la personeria fué promesas, qldo me iban a alyucke con arrendo, con la educación de los miña pero no me ayudaron en nada, en nack, por eso me toco metormo a trabajar con los señores qlas me levaron a la ca'ree.

$41-$ El gobserno no me ayu da to en na da, pur lo tanto lo qias hare por los sbup plazecias como yo, es mala, no sirue para nada, porglwo si a yu de ra, nosotoos lor que teniamos nuestro rancho, nuestras terras, galli nas, ganadito, comida, por la bendita querrá, estamos trejallo, judiots y encarcelados.

5- De la crisz roja y la tal atención Social, nunca medijaron nada ni me han dicto nada. no se qlito será eso.

46 
6-Al seños gobrer no solo le puedo decir, qla no nos olvide qu tambien somos colombianos y bien olvidados, que nada temiamos que ver con la guerra, q1ate solo somos victimas, y to dos hablan de nosotoos pobres, pero aqul como yo estamos muchas personas olvidadas en cambuches o en las carceles aguantan do de todo y lejos de nuestoos hivos. Eso qua va hacer vida. Esto es duro, muy duro, 4 nddie nos a yuda, nadie.

Blanoa luz diaz pap 36933249

47 
